\title{
Smart control of chemical gas sensors for the reduction of their time response
}

\author{
M. Dominguez-Pumar ${ }^{\mathrm{a}, *}$, L. Kowalski ${ }^{\mathrm{a}}$, R. Calavia ${ }^{\mathrm{b}}$, E. Llobet $^{\mathrm{b}}$ \\ ${ }^{a}$ Micro and Nano Technologies Group, Electronic Engineering Department, Universitat \\ Politècnica de Catalunya, Barcelona, Spain \\ ${ }^{b}$ MINOS-EMaS, Department dEnginyeria Electronica, Universitat Rovira i Virgili, \\ Tarragona, Spain
}

\begin{abstract}
The objective of this paper is to show the first results obtained with a gas sensor made of $\mathrm{Au}$-functionalized $\mathrm{WO}_{3}$ nanoneedles working under a closed-loop control designed to reduce its time response. The average temperature applied to the sensor is modulated to keep constant the average surface potential of the sensing nanostructures. This is done by periodically monitoring the resistivity of the sensing layer and generating temperature waveforms that enforce the condition: constant resistivity of the sensing layer at a reference temperature. Changes induced by the target gases must be compensated by changes in the average temperature being applied to the sensing layer. This signal, the average temperature applied to the sensor, is the new sensor output.
\end{abstract}

\section{Introduction}

The interest in gas sensors based on monocrystalline metallic oxides (nanoneedle, nanotubes and nanocolumns) has been increased in recent years. These nanomaterials present very good properties since most of their atoms are located on the surface and, therefore, can interact better with the environment, thus enhancing the sensitivity of the sensors. They also have a high crystallinity, thus reducing the presence of damages in the inter-grain boundaries, are more

\footnotetext{
${ }^{*}$ Corresponding author

Email address: manuel.dominguez@upc.edu (M. Dominguez-Pumar)
} 
stable than their polycrystalline counterparts and they present a well defined controllable surface (chemical composition and crystalline phase). On the other side, though, gas sensors based on these materials present a slow time response to changes in gas concentrations and may present unwanted long term drifts in their characteristics.

Measurements with this type of sensors are usually made in an 'open-loop' configuration. With this approach, the temperature of the sensing layer is set at the desired working point. Variations in gas concentrations generate changes in the concentration of the chemical species within the sensing layer, which result in changes in the resistivity of the sensing layer. Temperature modulations have been applied, thereby improving the selectivity of the sensors, but the time response still remains slow, [1. Extensive work has been done in the obtention of dynamic models, 2, 3]. It is widely accepted the necessity of using nonlinear models in order to describe the time evolution of the chemical reactions within the sensing layer. As a result, some works have used neural networks, [4, probabilistic state estimation, [5], reservoir computing, 6], or Support Vector Machines, [7].

The approach in this paper is to apply closed-loop control techniques that are often used in other fields, 8, 9, to control the average surface potential of the nanostructures. The control will keep this potential constant by adapting the average temperature profile of the sensing layer, which is the new output signal of the system. The final objective is to obtain a reduction of the time response of the sensor to changes in the target gases.

\section{Closed-loop control}

In order to design the control loop the first step is the analysis of the influence of temperature on the sensing layer. The nanoneedle conductance, according to potential barrier theory, [10, is:

$$
G=G_{0} T^{-\frac{3}{2}} e^{-\frac{q V_{S}}{k_{B} T}}
$$


where $G_{0}$ is a factor taking into account different parameters of the nanoneedle geometry and other electrical characteristics, $V_{S}$ is the average surface potential, $k_{B}$ is the Boltzmann constant and $T$ is temperature. Other conduction models (diffusion theory or thermoelectronic emission theory) generate equivalent expressions. In any case, the conductivity of the sensing layer is a function of temperature and the average surface potential.

The surface potential depends on the physisorption and chemisorption (and their inverse counterparts) of species within the surface of the sensing layer. The effective surface-charge density ultimately determining this surface potential, is the result of one or several chemical reactions taking place on the surface of the sensing layer. The surface potential is a function of the density of the occupied states and has the expression: $V_{s}=q N_{s}^{2} /\left(2 \epsilon_{r} \epsilon_{0} N_{d}\right)$, where $\epsilon_{r} \epsilon_{0}$ is the electrical permittivity of the semiconductor and $N_{s}$ the density of the occupied states and $N_{d}$ is the total density of surface states, [1].

This means that changes in temperature generate changes in the sensing layer by two different mechanisms:

- The first one involves the potential barrier theory (or other conduction theories) and is a very fast process. Sudden changes in temperature generate almost instantaneous changes in the resistivity of the sensing layer by way of the explicit temperature dependence in expression (1).

- The second one is due to changes in the surface potential, $V_{S}$. The effective surface-charge density depends on the dynamics of the intrinsic and extrinsic surface states, which depend on the target gases in contact with the surface as well as on temperature. All these processes have a very slow time evolution, 12 .

The objective of the proposed control is to keep constant the average surface potential of the sensing layer by changing its average temperature. Fast temperature commutations will be used to measure periodically the resistivity of the sensing layer at a constant reference temperature. The proposed control 


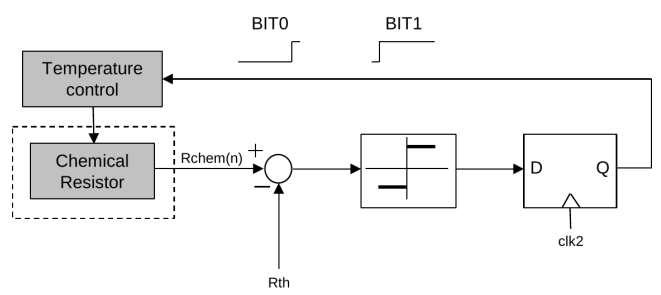

Figure 1: Chemical sigma-delta modulator topology. At each sampling time, depending on whether the chemical resistance, measured at the reference temperature $T_{\text {high }}$, is below (above) the desired value, a BIT1 (BIT0) temperature waveform is applied to the sensor.

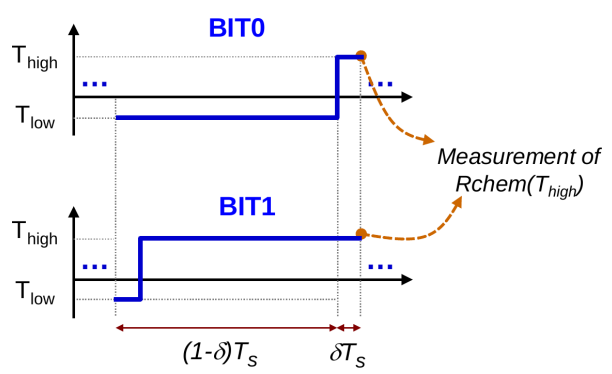

Figure 2: Temperature waveforms applied during each sampling period of the chemical control loop.

will generate temperature waveforms that in average will tend to keep constant this surface potential. The new output signal of the sensor is, therefore, the average temperature of the sensor. By suppressing the change in surface potential, and hence the slow dynamics associated to this change, we expect to improve significantly the dynamics of sensor response and recovery.

\section{Implementation}

The implementation that has been made includes two control loops. The first one is used to set the desired temperature of the sensing layer and is based on sigma-delta modulation, 8 . The topology of the second level control loop is shown in Figure 1. It is also a sampled system. At any given time two possible temperatures are applied to the sensing layer: $T_{\text {high }}$ or $T_{\text {low }}$. This is done by 
a)

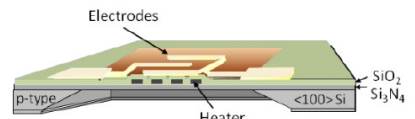

b)
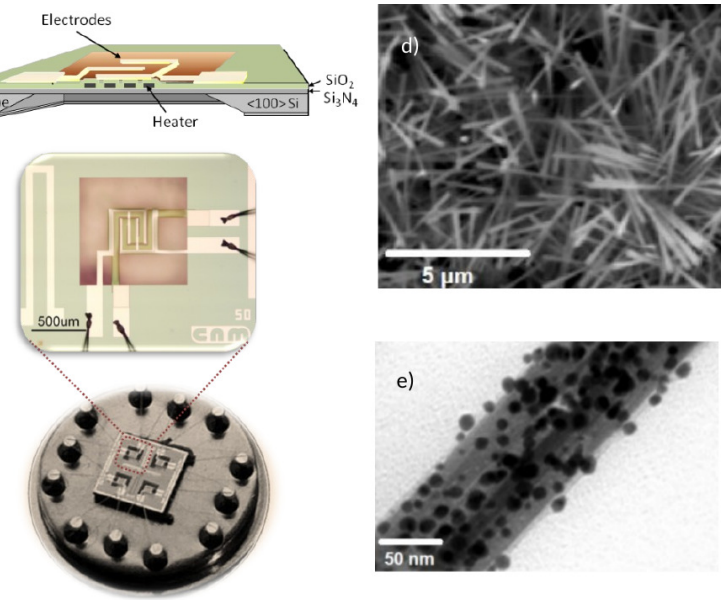

Figure 3: Drawing of a sensor (stack of interdigited Pt electrodes and polysilicon heating element sandwitched between silicon dioxide and silicon nitride layers (a); drawing of a sensor element seen from the top (b); a silicon chip comprises four micromachined sensors (c); SEM micrograph of CVD-grown tungsten oxide nanowires coating the electrode area (d); TEM image showing the $\mathrm{Au}$ nanoparticles coating a tungsten oxide nanowire.

adequately changing the threshold of the first temperature control loop.

In this feedback loop, the value of the chemical resistor is used to set up the actuation on the device during the next clock cycle. During each clock cycle, only two possible temperature profiles are applied to the device: BIT0 or BIT1 (shown in Figure 2). Both profiles have been designed to end at the same temperature, $T_{\text {high }}$, and the chemical resistance is measured at the end of each sampling period. With this approach, the feedback variable is the resistivity of the sensing layer measured at a constant temperature: $T_{\text {high }}$. In our experiments, the control loop feedback was designed for an oxidizing atmosphere and, therefore, the control increases (decreases) the average temperature if the resistivity of the sensing layer is below (above) the desired value, therefore applying a BIT1 (BIT0) temperature waveform during the next clock cycle. 


\section{Experimental results}

The gas MEMS microsensors used in this work have active layers made of nanoneedles of $\mathrm{WO}_{3}$ decorated with $\mathrm{Au}$ nanoparticles (see Figure 3). The $\mathrm{WO}_{3}$ nanoneedles functionalized with gold have been grown in a single step using localized aerosol-assisted chemical vapor deposition (AACVD), of tungsten hexacarbonyl (20 mg, W(CO) $)_{6}$, SigmaAldrich, $\geq 97 \%$ ) with tetrachloroauric acid trihydrate $\left(\left(1 \mathrm{mg}, \mathrm{HAuCl}_{4} \cdot 3 \mathrm{H}_{2} \mathrm{O}\right.\right.$, SigmaAldrich, 99.9\%). These active sensing layers were grown on top of silicon MEMS membranes that comprise a pair of interdigitated gold electrodes and an embedded polysilicon resistor that performs the function of sensor heater, [13, 14. The membrane has an area of $450 \times 450$ $\mu m^{2}$ and the interdigitated electrode gap is $50 \mu m$. The temperature coefficient of the polysilicon resistor is $6.79 \mathrm{e}-4 \mathrm{~K}^{-1}$. By continuously monitoring the value of this resistor it is possible to estimate the time evolution of the temperature of the membrane of the sensor.

The sensing layer consists of a mesh of randomly oriented, single crystalline, tungsten oxide nanoneedles decorated with gold nanoparticles. The diameter of nanoneedles ranges between 60 to $120 \mathrm{~nm}$ and their length is about $6 \mathrm{mi}$ crons. The average diameter of gold nanoparticles is $7 \mathrm{~nm}$. More details on the morphology and composition of the film can be found elsewhere, [15. The morphology of the sensing layer is shown in Figure 3 .

\subsection{Open loop characterization: fast and slow time scales}

The objective of this section is to experimentally analyze how a change in the average temperature of the sensing layer generates a change in its resistivity. To this effect several experiments have been perfomed on which the value of the temperature of the sensing layer, determined by the value of the polysilicon resistor, is switched between two possible values $\left(T_{\text {high }}\right.$ and $\left.T_{\text {low }}\right)$. In a first experiment the sensor is kept a $10 \%$ of the time at the $T_{\text {high }}$ and $90 \%$ at $T_{\text {low }}$. In a second experiment, the percentages are exchanged. In both cases, the repetition frequency of the pulses was $2 \mathrm{~Hz}$. The temperature actuation 


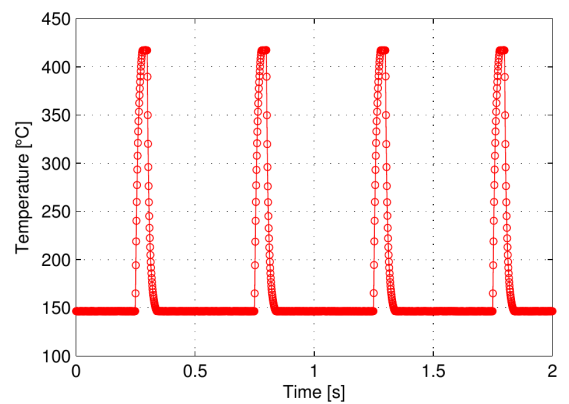

Figure 4: Temperature waveforms applied in the open loop experiment consisting of a constant sequence of BIT0 temperature waveforms (10\% of the time at $T_{\text {high }}$ and $90 \%$ of the time at $\left.T_{\text {low }}\right)$. The repetition period has been $2 \mathrm{~Hz}\left(T_{\text {low }}=143^{\circ} C, T_{\text {high }}=415^{\circ} C, I\left(R_{\text {chem }}\right)=\right.$ $3.5 \mu A)$.

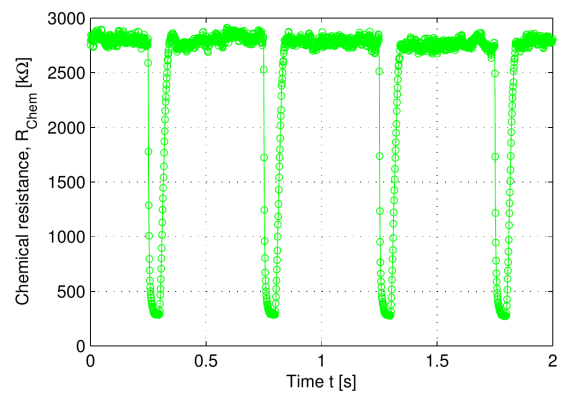

Figure 5: Time evolution of the chemical resistance for the experiment of Figure 4 Fast temperature changes generate fast changes in the chemical resistivity of the layer.

waveforms for the first case can be observed in Figure 4. The corresponding values taken by the chemical resistor can be observed in Figure 5 . As it is expected from the $\mathrm{n}$ semiconductor characteristic of the sensing layer, if the temperature is increased (decreased) the resistivity of the sensing layer suddenly decreases (increases). The dynamics of this process is very fast. Therefore, a fast sweep of the temperature of the sensing layer provides a curve showing the dependence on temperature of the chemical resistance, $R_{\text {chem }}(T)$, for an almost constant effective potential of the nanostructures.

This reflects the fact that the system presents dynamics with two time scales. 


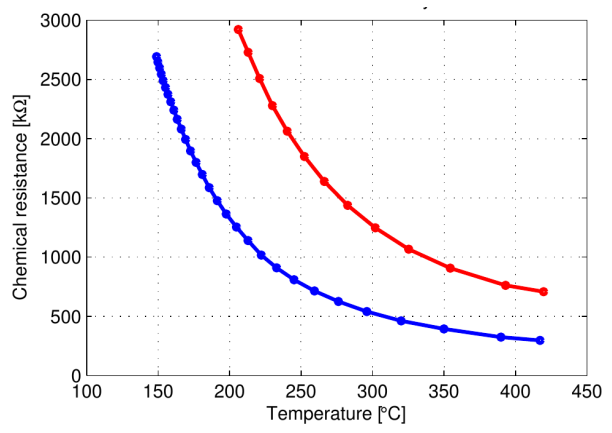

Figure 6: Plots of the chemical resistance as a function of the instantaneous temperature of the sensing layer, on the falling edge of the temperature waveform, $R_{\text {chem }}(T)$. Each point is the average of 100 cycles. Upper curve (in red): $R_{\text {chem }}(T)$ when a sequence of constant BIT1 waveforms has been applied for 120 minutes. Bottom curve (in blue): idem when the applied sequence consists only of BIT0 temperature waveforms. Higher average temperature profiles increase the values of $R_{\text {chem }}(T)\left(T_{\text {low }}=143^{\circ} C, T_{\text {high }}=415^{\circ} C, I\left(R_{\text {chem }}\right)=3.5 \mu A\right)$.

Increasing temperature reduces the resistivity of the semiconductor sensing layer in a process with fast dynamics. On the other hand, it enhances the chemisorption of oxygen species. This means that in the long term (slow time dynamics), it will increase the value of the $R_{\text {chem }}(T)$ curve. In order to observe this behaviour, an experiment has been performed on which the immediate and the long-term effects of the variation of temperature can be clearly observed. Figure 6 shows two sets of points. Those in the lower part of the graph (in blue) have been performed after applying during 2 hours the temperature profile is shown in Figure 4 10\% high temperature, 90\% low temperature. Those in the upper part of the graph (in red) have been performed after applying for 2 hours a complementary temperature profile ( $90 \%$ of the time high temperature, $10 \%$ low temperature). The continuous application of a temperature profile with a higher average temperature generates a vertical shift of the $R_{\text {chem }}(T)$ curve. This increase in $R_{\text {chem }}(T)$ can be associated to the temperature-driven increase in the number of adsorbed oxygen species on the n-type semiconductor tungsten oxide nanoneedles. 

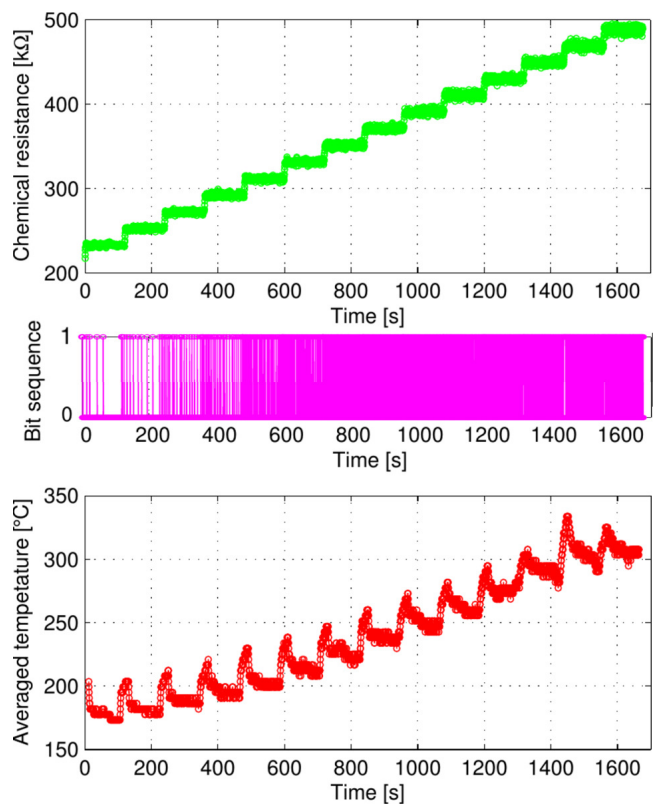

Figure 7: Top: curve of the chemical resistance, measured at $T_{\text {high }}$, as a function of time for an experiment on which the target resistance of the chemical loop is changed each 2 minutes from $250 \mathrm{k} \Omega$ in steps of $20 \mathrm{k} \Omega$. Middle: plot of the bit sequence applied during the experiment. Bottom: average temperature profile applied to the device during the experiment (average of 20 symbols per point). The duration of the BIT0 and BIT1 waveforms is $0.5 \mathrm{~s}$, and $\delta=0.1$ $\left(T_{\text {low }}=143^{\circ} C, T_{\text {high }}=415^{\circ} C, I\left(R_{\text {chem }}\right)=3.5 \mu A\right)$.

\subsection{Closed loop measurements}

An experiment has been carried out in order to analyze the capability of the control method to arbitrarily set the value of the chemical resistance (measured at a constant temperature, $\left.T_{\text {high }}\right)$. The set of admissible objective values for the chemical resistance is in fact provided by the maximum shift generated on the $R(T)$ curve (as seen in Figure 6), at the reference temperature at which the feedback is made. Once we select this temperature, the range for the threshold chemical resistor is found by looking at the crossing points with the top and bottom curves of Figure 6). Out of this range, the control will present saturation (a constant sequence of BIT0s or BIT1s).

Figure 7 a) shows an experiment on which fourteen different chemical resis- 


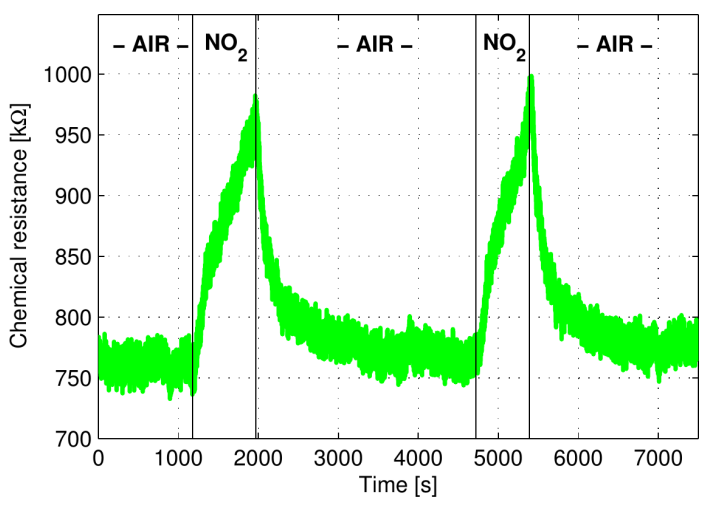

Figure 8: Sensor response operating at constant temperature, $T_{\text {high }}=415^{\circ} C$, when the sensor is exposed to synthetic air and $25 \mathrm{ppb}$ of $\mathrm{NO}_{2}$.

tance targets have been changed at intervals of 2 minutes, measured at $T_{\text {high }}$. There is a small settling time and after that the chemical resistance is kept around the assigned threshold value. Figure 7 .b) shows how the chemical bitstream changes as the chemical target values are changed. Finally Figure $7 \mathrm{c}$ ) shows the result of applying a low pass filter to the bitstream shown in Figure 7.b).

\subsection{Time response reduction of the transient response to changes in target gas concentration}

A first experiment was performed with the sensor working at a constant temperature, $T_{\text {high }}$, without control. As it can be observed in Figure 8 , the sensor response and recovery are very slow.

In the following experiment a target chemical resistance of $450 \mathrm{k} \Omega$ was set as the threshold value of the chemical control loop. This value is chosen to avoid saturation during the experiment. It must be taken into account that changes in the gas composition to which the sensor is exposed generate vertical shifts of the curves shown in Figure 6. If the sensor is exposed to an oxidizing gas, such as $\mathrm{NO}_{2}$, these curves will be displaced upwards. This means that once working in the closed-loop configuration, in order to keep constant the average surface 

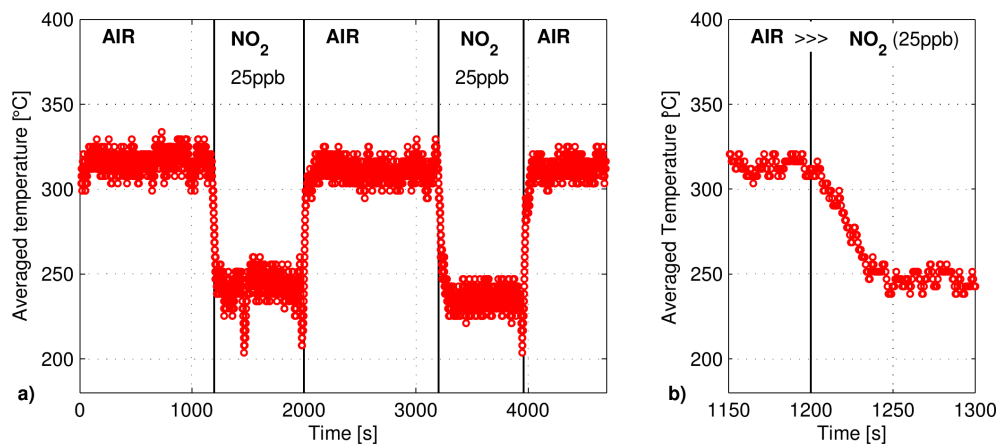

Figure 9: a) Average temperature applied to the device in an experiment on which the target chemical resistor was $450 \mathrm{k} \Omega, T_{\text {low }}=143^{\circ} C, T_{\text {high }}=415^{\circ} C, I\left(R_{\text {chem }}\right)=3.5 \mu \mathrm{A}$. The sensor was exposed for 20 minutes to synthetic air and 13 minutes to $25 \mathrm{ppb}$ of $\mathrm{NO}_{2}$. The cycling was repeated twice. Each average temperature is the average temperature of 50 consecutive temperature waveforms (either BIT0 or BIT1). The duration of the BIT0 and BIT1 waveforms is $0.5 \mathrm{~s}$, and $\delta=0.1$ b) Zoom of the first transition from synthetic air to $\mathrm{NO}_{2}$ in the same experiment.

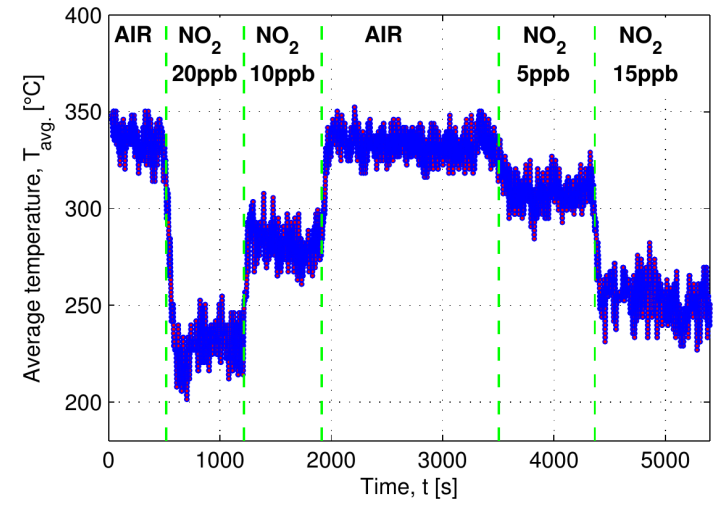

Figure 10: Average temperature applied to the device in an experiment on which the target chemical resistor was $560 \mathrm{k} \Omega, T_{\min }=125^{\circ} \mathrm{C}, T_{\max }=380^{\circ} \mathrm{C}, I\left(R_{\mathrm{chem}}\right)=16.2 \mu \mathrm{A}$. The sensor was exposed for different intervals of time to $0,5,10,15$ and $20 \mathrm{ppb}$ of $\mathrm{NO}_{2}$. Each average temperature is the average temperature of 120 consecutive temperature waveforms (either BIT0 or BIT1). The duration of the BIT0 and BIT1 waveforms is $0.5 \mathrm{~s}$, and $\delta=0.1$.

potential, the control loop will decrease the average temperature of the sensor (by generating more BIT0 waveforms). Therefore, in the absence of the target 
gas, it is interesting to set a target resistance such that the average number of BIT1 waveforms is around $90 \%$. Exposure to oxidizing gases will decrease this number and the limit is reached in saturation (absence of BIT1 waveforms), at which point the system would be in an open-loop configuration.

In the case of a reducing atmosphere, it would be interesting to set a target chemical resistance having a low average number of BIT1 waveforms (around $10 \%)$.

In the experiment of Figure 9, the sensor was in contact with synthetic air for 20 minutes, then in contact with $25 \mathrm{ppb}$ of $\mathrm{NO}_{2}$ for 13 minutes. The whole cycling was repeated two times. In order to set different target gas concentrations, a calibrated $\mathrm{NO}_{2}$ cylinder with air as balance gas was employed. This was further diluted by employing a computer controlled mass flow meter system and a cylinder of dry air. The total flow was kept constant at $200 \mathrm{ml} / \mathrm{min}$ throughout the measurement process. The dead volume within the gas sensor chamber was $4 \mathrm{ml}$. Under these conditions, the ambient in the measurement chamber is completely renewed in less than 2 seconds.

Figure 9 shows the average bitstream as a function of time of the chemical control loop. As it can be observed in order to keep constant the resistivity of the sensing layer it is necessary to decrease the average temperature. The transient response is fast (time response below the 50s). It must be noted that the recovery time is very similar to the response time, indicating a clear improvement of the sensor dynamics.

This experiment shows that in order to keep the sensor on the condition set by the closed loop it is necessary to change the average temperature of the sensing layer. From this signal it is possible to extract information on changes of the concentration of the target gases and in particular ameliorate the dynamics of baseline recovery. The sensitivity to different concentration levels of the target gas has been analyzed in another experiment using a second sensor with the same type of sensing layer. The results can be observed in Figure 10. The sensor is exposed to different concentrations of $\mathrm{NO}_{2}: 0,5,10,15$ and $20 \mathrm{ppb}$. As it can be observed the control loop generates different average temperatures 
in order to keep constant the target objective resistance measured at constant temperature.

\section{Conclusions}

The first experiments of the results of applying a closed-loop control to a gas sensor made of $\mathrm{WO}_{3}$ nanoneedles functionalized with $\mathrm{Au}$ nanoparticles has been shown. With the proposed control, changes in concentration of the target gases are compensated by changes in the average temperature applied to the sensor. This results in a clear improvement in the time response of the sensor. To the knowledge of the authors this is the first time that a closed loop control in chemical variables in a nanostructured sensing layer is applied to gas sensing.

\section{Acknowledgements}

We are indebted to Dr. Annanouch for growing the tungsten oxide nanoneedles on the micromachined substrates provided by Dr. Cané and Dr. Gracia. Funded in part by MINECO under grants num. TEC2013-48102-C2-1-P and TEC2012-32420. E.L. is supported by the Catalan Institution for Research and Advanced Studies via the ICREA Academia award.

\section{References}

[1] A. Vergara, R. Calavia, R. M. Vázquez, A. Mozalev, A. Abdelghani, R. Huerta, E. H. Hines, E. Llobet, Multifrequency interrogation of nanostructured gas sensor arrays: A tool for analyzing response kinetics, Anal. Chem. 84 (2012) 7502-7510.

[2] E. Llobet, J. Brezmes, X. Vilanova, J. E. Sueiras, X. Correig, Qualitative and quantitative analysis of volatile organic compounds using transient and steady-state responses of a thick-film tin oxide gas sensor array, Sensors Actuators B Chem. 41 (1997) 13-21. 
[3] C. Di Natale, S. Marco, F. Davide, A. D'Amico, Sensor-array calibration time reduction by dynamic modelling, Sensors Actuators B Chem. 25 (1995) 578-583.

[4] A. Pardo, S. Marco, J. Samitier, Nonlinear inverse dynamic models of gas sensing systems based on chemical sensor arrays for quantitative measurements, Instrum. Meas. IEEE Trans. 47 (1998) 644-651.

[5] R. Gosangi, R. Gutierrez-Osuna, Active temperature modulation of metaloxide sensors for quantitative analysis of gas mixtures, Sensors Actuators B Chem. 185 (2013) 201-210.

[6] J. Fonollosa, S. Sheik, R. Huerta, S. Marco, Reservoir computing compensates slow response of chemosensor arrays exposed to fast varying gas concentrations in continuous monitoring, Sensors Actuators B Chem. 215 (2015) 618-629.

[7] S. De Vito, A. Castaldo, F. Loffredo, E. Massera, T. Polichetti, I. Nasti, P. Vacca, L. Quercia, G. Di Francia, Gas concentration estimation in ternary mixtures with room temperature operating sensor array using tapped delay architectures, Sensors Actuators, B Chem. 124 (2007) 309316.

[8] M. Dominguez-Pumar, V. Jimenez, J. Ricart, L. Kowalski, J. Torres, S. Navarro, J. Romeral, L. Castañer, A hot film anemometer for the Martian atmosphere, Planet. Space Sci. 56 (2008) 1169-1179.

[9] M. Dominguez-Pumar, S. Gorreta, J. Pons-Nin, F. Gomez-Rodriguez, D.M. Gonzalez-Castano, M. Muschitiello, Closed-Loop Compensation of Dielectric Charge Induced by Ionizing Radiation, J. Microelectromechanical Syst. 24 (2015) 534-536.

[10] G. Tulzer, S. Baumgartner, E. Brunet, G. C. Mutinati, S. Steinhauer, A. Köck, P. E. Barbano, C. Heitzinger, Kinetic parameter estimation and 
fluctuation analysis of $\mathrm{CO}$ at $\mathrm{SnO} 2$ single nanowires, Nanotechnology 24 (2013) 315501.

[11] R. Ionescu, E. Llobet, S. Al-Khalifa, J. Gardner, X. Vilanova, J. Brezmes, $\mathrm{X}$. Correig, Response model for thermally modulated tin oxide-based microhotplate gas sensors, Sensors Actuators B Chem. 95 (2003) 203-211.

[12] J. Ding, T. J. McAvoy, R. E. Cavicchi, S. Semancik, Surface state trapping models for SnO2-based microhotplate sensors, Sensors Actuators B Chem. 77 (2001) 597-613.

[13] Fatima E. Annanouch, Zouhair Haddi, Stella Vallejos, Polona Umek, Peter Guttmann, Carla Bittencourt, and Eduard Llobet, Aerosol-assisted CVDgrown WO3 nanoneedles decorated with copper oxide nanoparticles for the selective and humidity-resilient detection of H2S., ACS Appl. Mater. Interfaces 7 (2015) 6842-51.

[14] S. V. F.E. Annanouch, I. Gràcia, E. Figueras, E. Llobet, C. Cané, Localized aerosol-assisted CVD of nanomaterials for the fabrication of monolithic gas sensor microarrays, Sensors Actuators B Chem. 216 (2015) 374-383.

[15] S. Vallejos, P. Umek, T. Stoycheva, F. Annanouch, E. Llobet, X. Correig, P. De Marco, C. Bittencourt, C. Blackman, Single-Step Deposition of Auand Pt-Nanoparticle-Functionalized Tungsten Oxide Nanoneedles Synthesized Via Aerosol-Assisted CVD, and Used for Fabrication of Selective Gas Microsensor Arrays, Adv. Funct. Mater. 23 (2013) 1313-1322. 